\title{
Harm Reduction Service Utilization Among People Who Inject Drugs in Tajikistan: Prevalence and Precipitating Factors
}

Mary M. Tate

Yale University School of Public Health

Daniel J. Bromberg

Yale University School of Public Health

Kamiar Alaei

California State University Long Beach College of Health and Human Services

\section{Saifuddin Karimov}

Ministry of Health and Protection of Population

\section{Dilshod Saidi}

Ministry of Health and Social Protection of Population

Arash Alaei ( $\square$ alaeiarash@gmail.com )

Institute for International Health and Education Albany, New York USA https://orcid.org/0000-00023302-6245

\section{Research article}

Keywords: Tajikistan , National AIDS Registry, positive deviancy

Posted Date: August 25th, 2020

DOI: https://doi.org/10.21203/rs.3.rs-50617/v1

License: (9) This work is licensed under a Creative Commons Attribution 4.0 International License. Read Full License 


\section{Abstract}

Tajikistan is in a unique geopolitical location along the global heroin trade route, exacerbating its own opioid use disorder and HIV epidemics. With one of the highest rates of opioid use disorder in the world, and 20,000-30,000 people who inject drugs in the country, Tajikistan's government and international actors have provided harm reduction measures for people who use drugs, like narcology centers, needle and syringe programs, and methadone maintenance therapy. No implementation science studies have been conducted in Tajikistan and the current implementation gaps in service uptake are unknown. The purpose of this paper is to determine the prevalence of harm reduction service uptake among people who use drugs in Tajikistan, and determine which factors are associated with service uptake. Methods This paper uses data from the National AIDS Registry, subset to patients who use drugs $(n=11,029)$ and crosssectional data from a bio-behavioral survey conducted in $2017(n=2,390)$. Univariate and multivariate logistic regression were used to assess associations between study variables and probably of uptake of narcology center registration, uptake of needle and syringe programs, and registration into methadone maintenance therapy. Results Fewer than half of all people who inject drugs (42.4\%) were registered with the narcology center, most people who inject drugs (88.6\%) reported always having access to clean syringes, and only $5.3 \%$ of PWID had ever engaged in methadone treatment in Tajikistan. There were ethnic differences in service uptake - with ethnic Russians and Uzbeks less likely to use services than ethnic Tajiks. Men who have sex with men and people living with HIV were also more likely to access services than heterosexual or seronegative individuals. Conclusion Narcology center registration and clean needle coverage are high in Tajikistan. Methadone maintenance therapy uptake, however, is low, like in other countries in Eastern Europe and Central Asia. NGO and government initiatives that target risk groups (like LGBTQ+ people and people living with HIV) seem to have been somewhat effective at recruiting their clientele into services. Future research might focus on the "positive deviancy" of these subgroups of people who use drugs to learn how to increase service uptake generally.

\section{Introduction}

Approximately $90 \%$ of the world's opium is produced in Afghanistan (1). The $1357 \mathrm{~km}$ border that stretches between Afghanistan and Tajikistan forms a critical stopping point in the so-called "Northern route", through which heroin is smuggled from Afghanistan to the Russian Federation and Europe (2). It is estimated that 118 metric tons of heroin are smuggled through the Northern route annually, emphasizing the importance of Tajikistan's location as a gateway between Afghanistan and Europe. Among seizures of trafficked drugs in Central Asia (approx. $10,000 \mathrm{~kg}$ is seized annually), $73 \%$ of total heroin seizures and $48 \%$ of total opium seizures occurred in Tajikistan (2).

Tajikistan's geographic position in the global heroin trade has had considerable impact on its own population as well. The annual prevalence of opioid use in Tajikistan is $0.52 \%$ among the adult population, giving it the 25th highest rate of opioid use in the world(3). There are approximately 20,00030,000 people who inject drugs (PWID) in Tajikistan, with heroin derivatives being the overwhelming majority of substances injected (4). 
Services for PWID in Tajikistan include narcological institutions that provide referral for specialized services, needle exchange programs, and opioid substitution therapy (OST). Of the 1152 inpatients at the narcological institutions across the country in $2008,84.2 \%$ were diagnosed as people who use heroin (5).

The publicly funded "narcological" institutions are a remnant of the Soviet drug treatment system that focused on medicalized detoxification and close control of people who use drugs (PWUD) (6).

Registration with a narcological institution is commonly the first step for a PWUD to receive relevant care in Tajikistan.

According to the WHO, needle and syringe exchange programs in Tajikistan "represent some of the best coverage in Central Asia" (7). Since 2014, 52 trust points have been initiated around the country, 24 are run by NGO's and 27 exist in conjunction with government-run AIDS centers. The "trust points" provide a range of integrated services including needle exchange, condoms, HIV counselling, HIV educational materials and referrals to HIV testing facilities. In 2013, 9202 PWIDs used the trust points for needle exchange services, resulting in approximately 70 needles per PWID in the country (7).

There are currently 6 opioid substitution therapy (OST) sites run out of narcology facilities in Tajikistan (8). OST programs began as pilots in 2009 , with each OST site having the capacity to treat approximately 100 people. In 2013, 516 patients received OST with an achieved retention rate of 40-70\% over 12 months (7). A qualitative study conducted among PWID in Tajikistan showed mistrust and misinformation surrounding methadone that effectively barred PWID from seeking OST (9).

There are several NGOs in Tajikistan leading harm reduction programs for PWID. Spin Plus, originally created as a crisis center specializing in providing services to female PWID, provides services to more than 2000 PWID from its locations in Dushanbe and Vahdat (10). The organization focuses on HIV prevention (including counseling and testing), needle exchange programs, OST self-help groups, and outreach and counselling to PWID. International organizations including the UNDP, Global Fund, Red Crescent Society, and PSI are also involved in projects for PWID who are living with HIV, particularly through their support of "trust points" around the country (10). Additionally, NGOs RAN and Aperon have also worked in Tajikistan to improve access to harm reduction services for PWID for HIV prevention.

In implementation science, a preliminary step in studying and explicating the implementation of evidencebased treatments is identifying "implementation gaps" (11). The goal of this paper is to determine uptake of narcology center registration, methadone treatment uptake, and use of clean syringes and determine which patient-level characteristics are associated with utilization of these harm reduction service in Tajikistan.

\section{Methods}

\section{Datasets}


This paper uses two datasets. The first is the National AIDS Registry, subset to PWID patients ( $\mathrm{n}=$ 11,029). The National AIDS Registry which collects data on all incident HIV cases in Tajikistan and follows them through treatment. Whenever a patient tests positive for HIV, they undergo a questionnaire administered through face-to-face interview by AIDS Center staff. They also complete routine intake forms and are given a medical exam by AIDS Center physicians. The interviews give information about a patient's sexual history, history of drug use, as well as other social and behavioral factors. Each patient has a unique identification number in the nation-wide database; therefore, follow up tests of CD4 and viral load are linked to each individual patient.

This paper also uses cross-sectional data from a bio-behavioral survey (BBS) conducted in 2017 ( $n=$ $2,390)$. The BBS uses snowball sampling to recruit PWID from all over Tajikistan. The data is collected using face-to-face interviewer administered questionnaires, in either the Tajik or Russian languages. After the questionnaire, the respondent is given an HIV $1 \frac{1}{2}$ STAT-PAK $B$ DIPSTICK rapid test to determine their HIV status.

\section{BBS Measures}

Participants were asked "Are you currently registered with a narcology center for injecting drug use?" and could respond either "yes" or "no". This question was not recategorized during analysis.

Participants were asked "In the last 6 months, how often did you use a new, sterile needle?" and the interviewer clarified for each participants that "by new, sterile needle we mean a needle never used by anyone before". Participants could respond: "always", "most of the time", "half of the time", "rarely", or "never". During analysis, responses were collapsed so that all responses that were not "always" but also were not missing were categorized as "not always".

Patients who had responded that they had indeed "ever received any drug treatment for injection drug use" were later further asked "what kind of treatment have you received?" with "maintenance with methadone" being one of the options. During analysis, participants who responded that they had ever undergone methadone maintenance were categorized as such, while all other participants (including those who had never received any form of treatment) were categorized as "never received methadone maintenance".

\section{Analysis}

To determine differences between characteristics of (HIV-positive) PWID and non-PWID, several statistical tests were conducted. For continuous variables that are normally distributed, these $p$-value correspond to a t-test. For continuous variables that are not normally distributed, MannWhitney $\mathrm{U}$ tests were performed. For categorical variables, $\mathrm{p}$-values correspond to a Pearson Chi-Square test.

Then, using the BBS survey data, we assessed correlates with the following OUD service uptake: 1) needle and syringe programs, 2) registration with the local service delivery point, 3) registration with a narcology 
center, 4) current engagement with methadone therapy. Both univariable and multivariable regression were conducted.

All analyses were conducted using the finalfit package (12) in RStudio(13).

\section{Results}

Among PLHIV, those that were from Gorno-Badakhshan and Dushanbe City were most likely to be PWID; men are much more likely to be PWID than women; and incarcerated people are more likely to be PWID. HIV-positive PWID are less likely to have had sex with female sex workers, or have ever had male-to-male sexual contact, than non-PWID. PWID are significantly more likely to present late with HIV (CD4 $<200$ at diagnosis), and are much more likely to have HCV, HBV, TB, and advanced HIV disease than non-PWID PLHIV (Table 1). 
Table 1

Summary descriptive table by groups of HIV-positive PWID and Non-PWID (National AIDS Registry Dataset)

\begin{tabular}{|c|c|c|c|}
\hline & $\begin{array}{l}\text { HIV-Positive Non- } \\
\text { PWID }\end{array}$ & $\begin{array}{l}\text { HIV-Positive } \\
\text { PWID }\end{array}$ & P-value \\
\hline & $N=7341$ & $N=3688$ & \\
\hline Region: & & & $<0.001$ \\
\hline Badakhshan & $272(3.71 \%)$ & $381(10.3 \%)$ & \\
\hline DRS & 1625 (22.1\%) & $595(16.1 \%)$ & \\
\hline Dushanbe & 1370 (18.7\%) & 1191 (32.3\%) & \\
\hline Khatlon & 2216 (30.2\%) & $841(22.8 \%)$ & \\
\hline Sughd & 1858 (25.3\%) & $680(18.4 \%)$ & \\
\hline Age: & & & $<0.001$ \\
\hline$<18$ & 1047 (14.3\%) & $1(0.03 \%)$ & \\
\hline $18-24$ & $259(3.53 \%)$ & $5(0.14 \%)$ & \\
\hline $25-34$ & 2016 (27.5\%) & $389(10.5 \%)$ & \\
\hline $35-44$ & 2377 (32.4\%) & 1597 (43.3\%) & \\
\hline $45-55$ & $1181(16.1 \%)$ & 1394 (37.8\%) & \\
\hline $55<$ & $461(6.28 \%)$ & $302(8.19 \%)$ & \\
\hline Gender: & & & $<0.001$ \\
\hline Male & 3575 (48.7\%) & 3527 (95.6\%) & \\
\hline Female & 3766 (51.3\%) & $161(4.37 \%)$ & \\
\hline Migrant: & & & $<0.001$ \\
\hline No & $5942(80.9 \%)$ & 3240 (87.9\%) & \\
\hline Yes & 1399 (19.1\%) & $448(12.1 \%)$ & \\
\hline Relationship Status: & & & $<0.001$ \\
\hline Single & $116(1.66 \%)$ & $50(1.41 \%)$ & \\
\hline Civil union & $73(1.05 \%)$ & $20(0.56 \%)$ & \\
\hline Divorced & $654(9.39 \%)$ & $585(16.5 \%)$ & \\
\hline Married & $4194(60.2 \%)$ & $1582(44.5 \%)$ & \\
\hline Other & $12(0.17 \%)$ & $2(0.06 \%)$ & \\
\hline
\end{tabular}




\begin{tabular}{|c|c|c|c|}
\hline & $\begin{array}{l}\text { HIV-Positive Non- } \\
\text { PWID }\end{array}$ & $\begin{array}{l}\text { HIV-Positive } \\
\text { PWID }\end{array}$ & P-value \\
\hline Unmarried & $1556(22.3 \%)$ & $1247(35.1 \%)$ & \\
\hline Widowed & $362(5.20 \%)$ & $70(1.97 \%)$ & \\
\hline Education: & & & $<0.001$ \\
\hline Elementary & $175(2.62 \%)$ & $12(0.35 \%)$ & \\
\hline High school & $4844(72.5 \%)$ & $2975(85.8 \%)$ & \\
\hline None & $732(11.0 \%)$ & $87(2.51 \%)$ & \\
\hline Specialized high school & $365(5.46 \%)$ & $243(7.01 \%)$ & \\
\hline Unfinished high school & $259(3.88 \%)$ & $62(1.79 \%)$ & \\
\hline Unfinished University & $80(1.20 \%)$ & $32(0.92 \%)$ & \\
\hline University Degree & $228(3.41 \%)$ & $55(1.59 \%)$ & \\
\hline Employment: & & & $<0.001$ \\
\hline Employed & $661(9.00 \%)$ & $119(3.23 \%)$ & \\
\hline Incarcerated & $31(0.42 \%)$ & $156(4.23 \%)$ & \\
\hline Military & $17(0.23 \%)$ & $0(0.00 \%)$ & \\
\hline None & $6235(84.9 \%)$ & $3384(91.8 \%)$ & \\
\hline Other & $71(0.97 \%)$ & $23(0.62 \%)$ & \\
\hline Retired & $24(0.33 \%)$ & $4(0.11 \%)$ & \\
\hline Student & $283(3.86 \%)$ & $1(0.03 \%)$ & \\
\hline Student (higher education) & $19(0.26 \%)$ & $1(0.03 \%)$ & \\
\hline Ever had Male-to-Male Sexual Contact: & & & $<0.001$ \\
\hline No & $3982(97.7 \%)$ & $3467(98.9 \%)$ & \\
\hline Yes & $95(2.33 \%)$ & $37(1.06 \%)$ & \\
\hline Baseline CD4 & $389(304)$ & $324(220)$ & $<0.001$ \\
\hline $\begin{array}{l}\text { Number of Sexual Partners in the Last } 12 \\
\text { Months }\end{array}$ & $2.79(3.50)$ & $1.51(1.45)$ & $<0.001$ \\
\hline Ever had Sex with Female Sex Worker: & & & $<0.001$ \\
\hline No & $3567(60.0 \%)$ & $1715(77.3 \%)$ & \\
\hline Yes & $2194(36.9 \%)$ & $393(17.7 \%)$ & \\
\hline
\end{tabular}




\begin{tabular}{|llll|}
\hline & $\begin{array}{l}\text { HIV-Positive Non- } \\
\text { PWID }\end{array}$ & $\begin{array}{l}\text { HIV-Positive } \\
\text { PWID }\end{array}$ & P-value \\
\hline HIV stage diagnosis: & & & $<0.001$ \\
\hline 1st Stage & $2500(36.9 \%)$ & $720(26.0 \%)$ & \\
\hline 2nd Stage & $1590(23.5 \%)$ & $561(20.3 \%)$ & \\
\hline 3rd Stage & $1574(23.3 \%)$ & $796(28.8 \%)$ \\
\hline 4th Stage & $1105(16.3 \%)$ & $688(24.9 \%)$ & \\
\hline
\end{tabular}

Fewer than half of all PWID (42.4\%) were registered with the narcology center, and men were significantly more likely to be registered than women in the univariate analysis, but not in the multivariable analysis. Ethnic Uzbeks and Russians are less likely to be registered than ethnic Tajiks (adjusted ORs 0.68 and 0.33 , respectively), as were respondents that were employed (adjusted OR 0.71). The longer that a PWID had been using drugs, the higher their likelihood of being registered with the narcology center. PLHIV, MSM, and formerly incarcerated PWID were several times more likely to be registered (adjusted ORs 4.14, $4.99,2.22$, respectively) (Tables 2 and 3 ). 
Table 2

Summary descriptive table by HIV Status (Biobehavioral Survey Dataset)

\begin{tabular}{|c|c|c|c|}
\hline & Negative & Positive & P-value \\
\hline & $N=2125$ & $N=265$ & \\
\hline Years as PWID & $8.37(6.28)$ & $11.6(6.53)$ & $<0.001$ \\
\hline Sex: & & & $<0.001$ \\
\hline Female & $89(4.19 \%)$ & $27(10.2 \%)$ & \\
\hline Male & $2036(95.8 \%)$ & $238(89.8 \%)$ & \\
\hline Ethnicity: & & & 0.380 \\
\hline Kyrgyz & $1(0.05 \%)$ & $0(0.00 \%)$ & \\
\hline Other & $7(0.33 \%)$ & $1(0.38 \%)$ & \\
\hline Russian & $91(4.28 \%)$ & $12(4.53 \%)$ & \\
\hline Tajik & $1602(75.4 \%)$ & $214(80.8 \%)$ & \\
\hline Tatar & $12(0.56 \%)$ & $2(0.75 \%)$ & \\
\hline Turkmen & $3(0.14 \%)$ & $0(0.00 \%)$ & \\
\hline Ukrainian & $1(0.05 \%)$ & $0(0.00 \%)$ & \\
\hline Uzbek & $408(19.2 \%)$ & $36(13.6 \%)$ & \\
\hline Education: & & & $<0.001$ \\
\hline Complete secondary & $1263(59.4 \%)$ & $182(68.7 \%)$ & \\
\hline Higher & $237(11.2 \%)$ & $19(7.17 \%)$ & \\
\hline Incomplete higher & $104(4.89 \%)$ & $8(3.02 \%)$ & \\
\hline No Education & $35(1.65 \%)$ & $11(4.15 \%)$ & \\
\hline Primary,incomplete secondary & $60(2.82 \%)$ & $11(4.15 \%)$ & \\
\hline Specialized secondary & $426(20.0 \%)$ & $34(12.8 \%)$ & \\
\hline Marital_Status: & & & 0.013 \\
\hline Divorced & $423(19.9 \%)$ & $68(25.7 \%)$ & \\
\hline Married (formal or civil marriage) & $1319(62.1 \%)$ & $146(55.1 \%)$ & \\
\hline No answer & $4(0.19 \%)$ & $1(0.38 \%)$ & \\
\hline Single, never married & $312(14.7 \%)$ & $34(12.8 \%)$ & \\
\hline
\end{tabular}




\begin{tabular}{|c|c|c|c|}
\hline & Negative & Positive & P-value \\
\hline Widowed & $67(3.15 \%)$ & $16(6.04 \%)$ & \\
\hline Occupation: & & & $<0.001$ \\
\hline Neither work nor study & $818(38.5 \%)$ & $136(51.3 \%)$ & \\
\hline No answer & $5(0.24 \%)$ & $2(0.75 \%)$ & \\
\hline Study & $7(0.33 \%)$ & $0(0.00 \%)$ & \\
\hline Work & $1278(60.1 \%)$ & $127(47.9 \%)$ & \\
\hline Work and study & $17(0.80 \%)$ & $0(0.00 \%)$ & \\
\hline \multicolumn{4}{|l|}{ Injection_Frequency: } \\
\hline $1-4$ times a month & $433(20.4 \%)$ & $49(18.5 \%)$ & \\
\hline 2 to 3 times a day & $507(23.9 \%)$ & $74(27.9 \%)$ & \\
\hline 2 to 7 times a week & $255(12.0 \%)$ & $27(10.2 \%)$ & \\
\hline 5 or more times a day & $85(4.00 \%)$ & $19(7.17 \%)$ & \\
\hline Less than once a month & $143(6.73 \%)$ & $27(10.2 \%)$ & \\
\hline No Answer & $1(0.05 \%)$ & $0(0.00 \%)$ & \\
\hline Once a day & $701(33.0 \%)$ & $69(26.0 \%)$ & \\
\hline Frequency_Ability_Recieve_Clean_Syringe: & & & $<0.001$ \\
\hline Don't Know & $42(1.98 \%)$ & $9(3.40 \%)$ & \\
\hline No Answer & $0(0.00 \%)$ & $1(0.38 \%)$ & \\
\hline Not Always & $175(8.24 \%)$ & $45(17.0 \%)$ & \\
\hline Yes & $1908(89.8 \%)$ & $210(79.2 \%)$ & \\
\hline \multicolumn{4}{|c|}{ Frequency of Clean Syringe Use (Last 6 months): } \\
\hline Always & $1704(80.2 \%)$ & $104(39.2 \%)$ & \\
\hline Don't Know & $48(2.26 \%)$ & $14(5.28 \%)$ & \\
\hline Half of the time & $24(1.13 \%)$ & $10(3.77 \%)$ & \\
\hline Most of the time & $334(15.7 \%)$ & $129(48.7 \%)$ & \\
\hline Never & $3(0.14 \%)$ & $1(0.38 \%)$ & \\
\hline Rarely & $12(0.56 \%)$ & $7(2.64 \%)$ & \\
\hline Registered_Narcology: & & & $<0.001$ \\
\hline
\end{tabular}




\begin{tabular}{|c|c|c|c|}
\hline & Negative & Positive & P-value \\
\hline Don't Know & $29(1.36 \%)$ & $0(0.00 \%)$ & \\
\hline No & $1285(60.5 \%)$ & $61(23.0 \%)$ & \\
\hline No Answer & $1(0.05 \%)$ & $0(0.00 \%)$ & \\
\hline Yes & $810(38.1 \%)$ & $204(77.0 \%)$ & \\
\hline Currently Recieving any OUD Treatment: & & & 0.006 \\
\hline No & $504(64.6 \%)$ & $82(52.2 \%)$ & \\
\hline No Answer & $1(0.13 \%)$ & $0(0.00 \%)$ & \\
\hline Yes & $275(35.3 \%)$ & $75(47.8 \%)$ & \\
\hline Ever_Incarcerated: & & & $<0.001$ \\
\hline No & $1600(75.3 \%)$ & $132(49.8 \%)$ & \\
\hline No Answer & $19(0.89 \%)$ & $0(0.00 \%)$ & \\
\hline Yes & $506(23.8 \%)$ & $133(50.2 \%)$ & \\
\hline Condom_Last_Sex: & & & $<0.001$ \\
\hline No & $1087(67.1 \%)$ & $62(38.5 \%)$ & \\
\hline No partner & $228(14.1 \%)$ & $13(8.07 \%)$ & \\
\hline Yes & $304(18.8 \%)$ & $86(53.4 \%)$ & \\
\hline Last_HIV_test: & & & $<0.001$ \\
\hline Don't Know & $25(1.58 \%)$ & $7(2.76 \%)$ & \\
\hline In the last $6-12$ months & $416(26.3 \%)$ & $17(6.69 \%)$ & \\
\hline In the last 6 months & $873(55.2 \%)$ & $142(55.9 \%)$ & \\
\hline More than 12 months ago & $268(16.9 \%)$ & $85(33.5 \%)$ & \\
\hline No Answer & $0(0.00 \%)$ & $3(1.18 \%)$ & \\
\hline New_HIV_Diagnosis: & & & $<0.001$ \\
\hline No & $4(17.4 \%)$ & $242(96.4 \%)$ & \\
\hline Yes & $19(82.6 \%)$ & $9(3.59 \%)$ & \\
\hline Registered for Services at Nearest Delivery Point: & & & $<0.001$ \\
\hline Don't Know & $7(0.33 \%)$ & $1(0.38 \%)$ & \\
\hline No & 1102 (51.9\%) & $74(27.9 \%)$ & \\
\hline
\end{tabular}




\begin{tabular}{|llll|}
\hline & Negative & Positive & P-value \\
\hline No Answer & $3(0.14 \%)$ & $0(0.00 \%)$ & \\
\hline Yes & $1013(47.7 \%)$ & $190(71.7 \%)$ & \\
MSM_ever: & & & 0.547 \\
\hline Don't Know & $1(0.05 \%)$ & $0(0.00 \%)$ & \\
No & $1989(97.7 \%)$ & $231(97.1 \%)$ & \\
Yes & $46(2.26 \%)$ & $7(2.94 \%)$ & \\
In_Prison_Inject: & & & 0.007 \\
\hline 99 & $0(0.00 \%)$ & $1(0.75 \%)$ & \\
\hline No & $312(61.7 \%)$ & $66(49.6 \%)$ & \\
Yes & $194(38.3 \%)$ & $66(49.6 \%)$ & \\
\hline
\end{tabular}


Table 3

Univariate and Multivariate Logistic Associations with Registration at Narcology Center $(n=2,390)$

\begin{tabular}{|c|c|c|c|c|c|}
\hline & & Unregistered & Registered & $\begin{array}{l}\text { OR } \\
\text { (univariable) }\end{array}$ & $\begin{array}{l}\text { OR } \\
\text { (multivariable) }\end{array}$ \\
\hline \multirow[t]{2}{*}{ Sex } & Female & $80(69.0)$ & $36(31.0)$ & - & - \\
\hline & Male & $1296(57.0)$ & $978(43.0)$ & $\begin{array}{l}1.68(1.13- \\
2.53, p= \\
0.012)\end{array}$ & $\begin{array}{l}1.45(0.91- \\
2.37, \mathrm{p}= \\
0.126)\end{array}$ \\
\hline \multirow[t]{8}{*}{ Ethnicity } & Tajik & 990 (54.5) & $826(45.5)$ & - & - \\
\hline & Other & $5(62.5)$ & 3 (37.5) & $\begin{array}{l}0.72(0.15- \\
2.94, p= \\
0.652)\end{array}$ & $\begin{array}{l}0.35(0.07- \\
1.62, \mathrm{p}= \\
0.176)\end{array}$ \\
\hline & Russian & $80(77.7)$ & $23(22.3)$ & $\begin{array}{l}0.34(0.21- \\
0.54, p< \\
0.001)\end{array}$ & $\begin{array}{l}0.33(0.19- \\
0.57, \mathrm{p}< \\
0.001)\end{array}$ \\
\hline & Tatar & $11(78.6)$ & $3(21.4)$ & $\begin{array}{l}0.33(0.07- \\
1.05, \mathrm{p}= \\
0.087)\end{array}$ & $\begin{array}{l}0.37(0.08- \\
1.31, \mathrm{p}= \\
0.155)\end{array}$ \\
\hline & Turkmen & $3(100.0)$ & & NA & NA \\
\hline & Uzbek & $287(64.6)$ & $157(35.4)$ & $\begin{array}{l}0.66(0.53- \\
0.81, p< \\
0.001)\end{array}$ & $\begin{array}{l}0.68(0.53- \\
0.87, p= \\
0.002)\end{array}$ \\
\hline & Kyrgyz & & $1(100.0)$ & NA & NA \\
\hline & Ukrainian & & $1(100.0)$ & NA & NA \\
\hline \multirow[t]{6}{*}{ Education } & $\begin{array}{l}\text { Complete } \\
\text { secondary }\end{array}$ & 846 (58.5) & $599(41.5)$ & - & - \\
\hline & Higher & 135 (52.7) & $121(47.3)$ & $\begin{array}{l}1.27(0.97- \\
1.65, \mathrm{p}= \\
0.083)\end{array}$ & $\begin{array}{l}1.43(1.06- \\
1.93, p= \\
0.018)\end{array}$ \\
\hline & $\begin{array}{l}\text { Incomplete } \\
\text { higher }\end{array}$ & $76(67.9)$ & $36(32.1)$ & $\begin{array}{l}0.67(0.44- \\
1.00, p= \\
0.055)\end{array}$ & $\begin{array}{l}0.72(0.45- \\
1.14, \mathrm{p}= \\
0.166)\end{array}$ \\
\hline & No Education & $17(37.0)$ & $29(63.0)$ & $\begin{array}{l}2.41(1.33- \\
4.51, p= \\
0.005)\end{array}$ & $\begin{array}{l}1.91(0.97- \\
3.84, \mathrm{p}= \\
0.064)\end{array}$ \\
\hline & $\begin{array}{l}\text { Primary/ } \\
\text { Incomplete } \\
\text { Secondary }\end{array}$ & $41(57.7)$ & $30(42.3)$ & $\begin{array}{l}1.03(0.63- \\
1.67, \mathrm{p}= \\
0.894)\end{array}$ & $\begin{array}{l}1.02(0.59- \\
1.75, \mathrm{p}= \\
0.940)\end{array}$ \\
\hline & $\begin{array}{l}\text { Specialized } \\
\text { secondary }\end{array}$ & $261(56.7)$ & 199 (43.3) & $\begin{array}{l}1.08(0.87- \\
1.33, \mathrm{p}= \\
0.494)\end{array}$ & $\begin{array}{l}1.18(0.93- \\
1.49, \mathrm{p}= \\
0.182)\end{array}$ \\
\hline
\end{tabular}




\begin{tabular}{|c|c|c|c|c|c|}
\hline & & Unregistered & Registered & $\begin{array}{l}\text { OR } \\
\text { (univariable) }\end{array}$ & $\begin{array}{l}\text { OR } \\
\text { (multivariable) }\end{array}$ \\
\hline \multirow[t]{5}{*}{ Marital Status } & Divorced & 273 (55.6) & $218(44.4)$ & - & - \\
\hline & Married & $841(57.4)$ & $624(42.6)$ & $\begin{array}{l}0.93(0.76- \\
1.14, p= \\
0.484)\end{array}$ & $\begin{array}{l}1.09(0.86- \\
1.38, \mathrm{p}= \\
0.489)\end{array}$ \\
\hline & No answer & $4(80.0)$ & $1(20.0)$ & $\begin{array}{l}0.31(0.02- \\
2.14, p= \\
0.301)\end{array}$ & $\begin{array}{l}0.37(0.02- \\
3.35, \mathrm{p}= \\
0.438)\end{array}$ \\
\hline & $\begin{array}{l}\text { Single, never } \\
\text { married }\end{array}$ & 219 (63.3) & $127(36.7)$ & $\begin{array}{l}0.73(0.55- \\
0.96, p= \\
0.026)\end{array}$ & $\begin{array}{l}0.96(0.69- \\
1.34, \mathrm{p}= \\
0.824)\end{array}$ \\
\hline & Widowed & $39(47.0)$ & $44(53.0)$ & $\begin{array}{l}1.41(0.89- \\
2.26, \mathrm{p}= \\
0.146)\end{array}$ & $\begin{array}{l}1.15(0.66- \\
1.99, \mathrm{p}= \\
0.628)\end{array}$ \\
\hline \multirow[t]{5}{*}{ Occupation } & $\begin{array}{l}\text { Neither work } \\
\text { nor study }\end{array}$ & $477(50.0)$ & $477(50.0)$ & - & - \\
\hline & No answer & $5(71.4)$ & $2(28.6)$ & $\begin{array}{l}0.40(0.06- \\
1.87, \mathrm{p}= \\
0.275)\end{array}$ & $\begin{array}{l}0.38(0.04- \\
2.63, \mathrm{p}= \\
0.329)\end{array}$ \\
\hline & Study & 6 (85.7) & 1 (14.3) & $\begin{array}{l}0.17(0.01- \\
0.98, p= \\
0.098)\end{array}$ & $\begin{array}{l}0.21(0.01- \\
1.64, p= \\
0.198)\end{array}$ \\
\hline & Work & $874(62.2)$ & $531(37.8)$ & $\begin{array}{l}0.61(0.51- \\
0.72, p< \\
0.001)\end{array}$ & $\begin{array}{l}0.71(0.58- \\
0.86, \mathrm{p}< \\
0.001)\end{array}$ \\
\hline & Work and study & $14(82.4)$ & $3(17.6)$ & $\begin{array}{l}0.21(0.05- \\
0.66, p= \\
0.016)\end{array}$ & $\begin{array}{l}0.35(0.08- \\
1.16, p= \\
0.119)\end{array}$ \\
\hline \multirow[t]{2}{*}{ HIV Status } & Negative & 1315 (61.9) & $810(38.1)$ & - & - \\
\hline & Positive & $61(23.0)$ & $204(77.0)$ & $\begin{array}{l}5.43(4.05- \\
7.38, p< \\
0.001)\end{array}$ & $\begin{array}{l}4.14(3.00- \\
5.80, \mathrm{p}< \\
0.001)\end{array}$ \\
\hline Years as PWID & Mean (SD) & $7.1(5.4)$ & $10.9(7.0)$ & $\begin{array}{l}1.10(1.09- \\
1.12, p< \\
0.001)\end{array}$ & $\begin{array}{l}1.09(1.07- \\
1.10, p< \\
0.001)\end{array}$ \\
\hline \multirow[t]{2}{*}{ Ever Incarcerated } & No & $1126(65.0)$ & $606(35.0)$ & - & - \\
\hline & Yes & $231(36.2)$ & $408(63.8)$ & $\begin{array}{l}3.28(2.72- \\
3.97, \mathrm{p}< \\
0.001)\end{array}$ & $\begin{array}{l}2.22(1.79- \\
2.75, p< \\
0.001)\end{array}$ \\
\hline
\end{tabular}




\begin{tabular}{|c|c|c|c|c|c|}
\hline & & Unregistered & Registered & $\begin{array}{l}\text { OR } \\
\text { (univariable) }\end{array}$ & $\begin{array}{l}\text { OR } \\
\text { (multivariable) }\end{array}$ \\
\hline \multirow{2}{*}{$\begin{array}{l}\text { Ever had Male-to- } \\
\text { Male Sexual } \\
\text { contact }\end{array}$} & No & $1365(58.4)$ & $972(41.6)$ & - & - \\
\hline & Yes & $11(20.8)$ & $42(79.2)$ & $\begin{array}{l}5.36(2.85- \\
11.00, p< \\
0.001)\end{array}$ & $\begin{array}{l}4.99(2.40- \\
11.23, \mathrm{p}< \\
0.001)\end{array}$ \\
\hline
\end{tabular}

Only 5.3\% of PWID had ever engaged in methadone treatment in Tajikistan. Ethnic minorities, like Uzbeks and Russians, were significantly less likely to be engaged in methadone therapy than ethnic Tajiks. The longer that a PWID had been using drugs, the higher their likelihood of being enrolled in methadone therapy. While PWID enrolled in methadone therapy had a mean injection career of 11.7 years, those that were not enrolled had a mean injection career of 8.6 years. Both MSM and PLHIV were significantly more likely (Adjusted ORs: 3.37 and 3.86, respectively) to be engaged in methadone treatment than non-MSM and people who were HIV negative (Tables 2 and 4). 
Table 4

Univariable and Multivariable Logistic Associations with Enrollment in Methadone Therapy for Opioid Use Disorder $(n=2,390)$

\begin{tabular}{|c|c|c|c|c|c|}
\hline & & 0 & 1 & OR (univariable) & $\begin{array}{l}\text { OR } \\
\text { (multivariable) }\end{array}$ \\
\hline \multirow[t]{2}{*}{ Sex } & Female & $\begin{array}{l}109 \\
(94.0)\end{array}$ & $\begin{array}{l}7 \\
(6.0)\end{array}$ & - & - \\
\hline & Male & $\begin{array}{l}2155 \\
(94.8)\end{array}$ & $\begin{array}{l}119 \\
(5.2)\end{array}$ & $\begin{array}{l}0.86(0.42- \\
2.07, \mathrm{p}=0.707)\end{array}$ & $\begin{array}{l}0.72(0.31-1.94 \\
p=0.473)\end{array}$ \\
\hline \multirow[t]{8}{*}{ Ethnicity } & Tajik & $\begin{array}{l}1703 \\
(93.8)\end{array}$ & $\begin{array}{l}113 \\
(6.2)\end{array}$ & - & - \\
\hline & Kyrgyz & $\begin{array}{l}1 \\
(100.0)\end{array}$ & & NA & NA \\
\hline & Other & $\begin{array}{l}8 \\
(100.0)\end{array}$ & & NA & NA \\
\hline & Russian & $\begin{array}{l}102 \\
(99.0)\end{array}$ & $\begin{array}{l}1 \\
(1.0)\end{array}$ & $\begin{array}{l}0.15(0.01- \\
0.67, \mathrm{p}=0.058)\end{array}$ & $\begin{array}{l}0.13(0.01-0.68 \\
p=0.054)\end{array}$ \\
\hline & Tatar & $\begin{array}{l}12 \\
(85.7)\end{array}$ & $\begin{array}{l}2 \\
(14.3)\end{array}$ & $\begin{array}{l}2.51(0.39- \\
9.36, p=0.232)\end{array}$ & $\begin{array}{l}3.10(0.40- \\
14.25, p=0.199)\end{array}$ \\
\hline & Turkmen & $\begin{array}{l}3 \\
(100.0)\end{array}$ & & NA & NA \\
\hline & Ukrainian & $\begin{array}{l}1 \\
(100.0)\end{array}$ & & NA & NA \\
\hline & Uzbek & $\begin{array}{l}434 \\
(97.7)\end{array}$ & $\begin{array}{l}10 \\
(2.3)\end{array}$ & $\begin{array}{l}0.35(0.17- \\
0.64, \mathrm{p}=0.002)\end{array}$ & $\begin{array}{l}0.43(0.20-0.80 \\
p=0.014)\end{array}$ \\
\hline \multirow[t]{6}{*}{ Education } & Complete secondary & $\begin{array}{l}1369 \\
(94.7)\end{array}$ & $\begin{array}{l}76 \\
(5.3)\end{array}$ & - & - \\
\hline & Higher & $\begin{array}{l}235 \\
(91.8)\end{array}$ & $\begin{array}{l}21 \\
(8.2)\end{array}$ & $\begin{array}{l}1.61(0.95- \\
2.61, p=0.063)\end{array}$ & $\begin{array}{l}1.79(1.03-3.02 \\
p=0.033)\end{array}$ \\
\hline & Incomplete higher & $\begin{array}{l}107 \\
(95.5)\end{array}$ & $\begin{array}{l}5 \\
(4.5)\end{array}$ & $\begin{array}{l}0.84(0.29- \\
1.93, p=0.715)\end{array}$ & $\begin{array}{l}1.12(0.38-2.69 \\
p=0.815)\end{array}$ \\
\hline & No Education & $\begin{array}{l}42 \\
(91.3)\end{array}$ & $\begin{array}{l}4 \\
(8.7)\end{array}$ & $\begin{array}{l}1.72(0.51- \\
4.38, p=0.314)\end{array}$ & $\begin{array}{l}1.02(0.29-2.82 \\
p=0.968)\end{array}$ \\
\hline & $\begin{array}{l}\text { Primary,incomplete } \\
\text { secondary }\end{array}$ & $\begin{array}{l}70 \\
(98.6)\end{array}$ & $\begin{array}{l}1 \\
(1.4)\end{array}$ & $\begin{array}{l}0.26(0.01- \\
1.19, p=0.180)\end{array}$ & $\begin{array}{l}0.23(0.01-1.09 \\
p=0.149)\end{array}$ \\
\hline & $\begin{array}{l}\text { Specialized } \\
\text { secondary }\end{array}$ & $\begin{array}{l}441 \\
(95.9)\end{array}$ & $\begin{array}{l}19 \\
(4.1)\end{array}$ & $\begin{array}{l}0.78(0.45- \\
1.27, p=0.334)\end{array}$ & $\begin{array}{l}0.91(0.52-1.53 \\
p=0.734)\end{array}$ \\
\hline Marital_Status & Divorced & $\begin{array}{l}465 \\
(94.7)\end{array}$ & $\begin{array}{l}26 \\
(5.3)\end{array}$ & - & - \\
\hline
\end{tabular}




\begin{tabular}{|c|c|c|c|c|c|}
\hline & & 0 & 1 & OR (univariable) & $\begin{array}{l}\text { OR } \\
\text { (multivariable) }\end{array}$ \\
\hline & $\begin{array}{l}\text { Married (formal or } \\
\text { civil marriage) }\end{array}$ & $\begin{array}{l}1395 \\
(95.2)\end{array}$ & $\begin{array}{l}70 \\
(4.8)\end{array}$ & $\begin{array}{l}0.90(0.57- \\
1.45, p=0.646)\end{array}$ & $\begin{array}{l}1.19(0.73-1.98, \\
p=0.493)\end{array}$ \\
\hline & No answer & $\begin{array}{l}5 \\
(100.0)\end{array}$ & & NA & NA \\
\hline & Single, never married & $\begin{array}{l}322 \\
(93.1)\end{array}$ & $\begin{array}{l}24 \\
(6.9)\end{array}$ & $\begin{array}{l}1.33(0.75- \\
2.37, p=0.325)\end{array}$ & $\begin{array}{l}1.63(0.86-3.06, \\
p=0.131)\end{array}$ \\
\hline & Widowed & $\begin{array}{l}77 \\
(92.8)\end{array}$ & $\begin{array}{l}6 \\
(7.2)\end{array}$ & $\begin{array}{l}1.39(0.51- \\
3.29, p=0.479)\end{array}$ & $\begin{array}{l}1.16(0.40-2.96, \\
p=0.769)\end{array}$ \\
\hline \multirow[t]{5}{*}{ Occupation } & $\begin{array}{l}\text { Neither work nor } \\
\text { study }\end{array}$ & $\begin{array}{l}877 \\
(91.9)\end{array}$ & $\begin{array}{l}77 \\
(8.1)\end{array}$ & - & - \\
\hline & No answer & $\begin{array}{l}7 \\
(100.0)\end{array}$ & & NA & NA \\
\hline & Study & $\begin{array}{l}7 \\
(100.0)\end{array}$ & & NA & NA \\
\hline & Work & $\begin{array}{l}1356 \\
(96.5)\end{array}$ & $\begin{array}{l}49 \\
(3.5)\end{array}$ & $\begin{array}{l}0.41(0.28- \\
0.59, \mathrm{p}<0.001)\end{array}$ & $\begin{array}{l}0.53(0.35-0.78, \\
p=0.002)\end{array}$ \\
\hline & Work and study & $\begin{array}{l}17 \\
(100.0)\end{array}$ & & NA & NA \\
\hline \multirow[t]{2}{*}{ HIV_Status } & Negative & $\begin{array}{l}2043 \\
(96.1)\end{array}$ & $\begin{array}{l}82 \\
(3.9)\end{array}$ & - & - \\
\hline & Positive & $\begin{array}{l}221 \\
(83.4)\end{array}$ & $\begin{array}{l}44 \\
(16.6)\end{array}$ & $\begin{array}{l}4.96(3.33- \\
7.30, p<0.001)\end{array}$ & $\begin{array}{l}3.86(2.51-5.88, \\
p<0.001)\end{array}$ \\
\hline Years_as_PWID & Mean (SD) & $\begin{array}{l}8.6 \\
(6.3)\end{array}$ & $\begin{array}{l}11.7 \\
(6.9)\end{array}$ & $\begin{array}{l}1.06(1.04- \\
1.09, p<0.001)\end{array}$ & $\begin{array}{l}1.04(1.01-1.07 \\
p=0.006)\end{array}$ \\
\hline \multirow[t]{2}{*}{ Ever_Incarcerated } & No & $\begin{array}{l}1667 \\
(96.2)\end{array}$ & $\begin{array}{l}65 \\
(3.8)\end{array}$ & - & - \\
\hline & Yes & $\begin{array}{l}578 \\
(90.5)\end{array}$ & $\begin{array}{l}61 \\
(9.5)\end{array}$ & $\begin{array}{l}2.71(1.88- \\
3.89, p<0.001)\end{array}$ & $\begin{array}{l}1.81(1.20-2.73 \\
p=0.005)\end{array}$ \\
\hline \multirow[t]{2}{*}{ MSM_ever } & No & $\begin{array}{l}2221 \\
(95.0)\end{array}$ & $\begin{array}{l}116 \\
(5.0)\end{array}$ & - & - \\
\hline & Yes & $\begin{array}{l}43 \\
(81.1)\end{array}$ & $\begin{array}{l}10 \\
(18.9)\end{array}$ & $\begin{array}{l}4.45(2.07- \\
8.74, \mathrm{p}<0.001)\end{array}$ & $\begin{array}{l}3.37(1.46-7.20, \\
p=0.003)\end{array}$ \\
\hline
\end{tabular}


Table 5

Univariate and Multivariable Logistic Associations with Likelihood of "Always Having Access to Clean Syringes" $(\mathrm{n}=2,390)$.

\begin{tabular}{|c|c|c|c|c|c|}
\hline Dependent: & & No & Yes & $\begin{array}{l}\text { OR } \\
\text { (univariable) }\end{array}$ & $\begin{array}{l}\text { OR } \\
\text { (multivariable) }\end{array}$ \\
\hline \multirow[t]{2}{*}{ Sex } & Female & $\begin{array}{l}13 \\
(11.2)\end{array}$ & $\begin{array}{l}103 \\
(88.8)\end{array}$ & - & - \\
\hline & Male & $\begin{array}{l}259 \\
(11.4)\end{array}$ & $\begin{array}{l}2015 \\
(88.6)\end{array}$ & $\begin{array}{l}0.98(0.52- \\
1.71, p=0.952)\end{array}$ & $\begin{array}{l}0.74(0.38- \\
1.35, p=0.353)\end{array}$ \\
\hline \multirow[t]{8}{*}{ Ethnicity } & Tajik & $\begin{array}{l}195 \\
(10.7)\end{array}$ & $\begin{array}{l}1621 \\
(89.3)\end{array}$ & - & - \\
\hline & Other & $\begin{array}{l}1 \\
(12.5)\end{array}$ & $\begin{array}{l}7 \\
(87.5)\end{array}$ & $\begin{array}{l}0.84(0.15- \\
15.78, p= \\
0.873)\end{array}$ & $\begin{array}{l}0.68(0.11- \\
13.25, p= \\
0.728)\end{array}$ \\
\hline & Russian & $\begin{array}{l}13 \\
(12.6)\end{array}$ & $\begin{array}{l}90 \\
(87.4)\end{array}$ & $\begin{array}{l}0.83(0.47- \\
1.59, p=0.550)\end{array}$ & $\begin{array}{l}0.99(0.52-2.00 \\
p=0.966)\end{array}$ \\
\hline & Tatar & $1(7.1)$ & $\begin{array}{l}13 \\
(92.9)\end{array}$ & $\begin{array}{l}1.56(0.31- \\
28.49, p= \\
0.667)\end{array}$ & $\begin{array}{l}1.69(0.32- \\
31.19, p= \\
0.620)\end{array}$ \\
\hline & Ukrainian & $\begin{array}{l}1 \\
(100.0)\end{array}$ & & NA & NA \\
\hline & Uzbek & $\begin{array}{l}61 \\
(13.7)\end{array}$ & $\begin{array}{l}383 \\
(86.3)\end{array}$ & $\begin{array}{l}0.76(0.56- \\
1.03, \mathrm{p}=0.074)\end{array}$ & $\begin{array}{l}0.71(0.52- \\
0.98, p=0.036)\end{array}$ \\
\hline & Kyrgyz & & $\begin{array}{l}1 \\
(100.0)\end{array}$ & NA & NA \\
\hline & Turkmen & & $\begin{array}{l}3 \\
(100.0)\end{array}$ & NA & NA \\
\hline \multirow[t]{6}{*}{ Education } & $\begin{array}{l}\text { Complete } \\
\text { secondary }\end{array}$ & $\begin{array}{l}186 \\
(12.9)\end{array}$ & $\begin{array}{l}1259 \\
(87.1)\end{array}$ & - & - \\
\hline & Higher & $9(3.5)$ & $\begin{array}{l}247 \\
(96.5)\end{array}$ & $\begin{array}{l}4.05(2.17- \\
8.64, p<0.001)\end{array}$ & $\begin{array}{l}3.45(1.83- \\
7.40, p<0.001)\end{array}$ \\
\hline & Incomplete higher & $\begin{array}{l}11 \\
(9.8)\end{array}$ & $\begin{array}{l}101 \\
(90.2)\end{array}$ & $\begin{array}{l}1.36(0.75- \\
2.72, p=0.351)\end{array}$ & $\begin{array}{l}1.25(0.68- \\
2.54, p=0.504)\end{array}$ \\
\hline & No Education & $\begin{array}{l}8 \\
(17.4)\end{array}$ & $\begin{array}{l}38 \\
(82.6)\end{array}$ & $\begin{array}{l}0.70(0.34- \\
1.64, p=0.372)\end{array}$ & $\begin{array}{l}0.67(0.31- \\
1.61, p=0.335)\end{array}$ \\
\hline & $\begin{array}{l}\text { Primary,incomplete } \\
\text { secondary }\end{array}$ & $\begin{array}{l}12 \\
(16.9)\end{array}$ & $\begin{array}{l}59 \\
(83.1)\end{array}$ & $\begin{array}{l}0.73(0.40- \\
1.44, p=0.327)\end{array}$ & $\begin{array}{l}0.70(0.37- \\
1.41, p=0.287)\end{array}$ \\
\hline & $\begin{array}{l}\text { Specialized } \\
\text { secondary }\end{array}$ & $\begin{array}{l}46 \\
(10.0)\end{array}$ & $\begin{array}{l}414 \\
(90.0)\end{array}$ & $\begin{array}{l}1.33(0.95- \\
1.89, p=0.102)\end{array}$ & $\begin{array}{l}1.22(0.87- \\
1.75, p=0.263)\end{array}$ \\
\hline
\end{tabular}




\begin{tabular}{|c|c|c|c|c|c|}
\hline Dependent: & & No & Yes & $\begin{array}{l}\text { OR } \\
\text { (univariable) }\end{array}$ & $\begin{array}{l}\text { OR } \\
\text { (multivariable) }\end{array}$ \\
\hline \multirow[t]{5}{*}{ Marital_Status } & Divorced & $\begin{array}{l}91 \\
(18.5)\end{array}$ & $\begin{array}{l}400 \\
(81.5)\end{array}$ & - & - \\
\hline & $\begin{array}{l}\text { Married (formal or } \\
\text { civil marriage) }\end{array}$ & $\begin{array}{l}145 \\
(9.9)\end{array}$ & $\begin{array}{l}1320 \\
(90.1)\end{array}$ & $\begin{array}{l}2.07(1.55- \\
2.75, \mathrm{p}<0.001)\end{array}$ & $\begin{array}{l}2.09(1.55- \\
2.82, \mathrm{p}<0.001)\end{array}$ \\
\hline & No answer & $\begin{array}{l}1 \\
(20.0)\end{array}$ & $\begin{array}{l}4 \\
(80.0)\end{array}$ & $\begin{array}{l}0.91(0.13- \\
17.91, p= \\
0.933)\end{array}$ & $\begin{array}{l}1.16(0.16- \\
23.49, p= \\
0.895)\end{array}$ \\
\hline & $\begin{array}{l}\text { Single, never } \\
\text { married }\end{array}$ & $\begin{array}{l}21 \\
(6.1)\end{array}$ & $\begin{array}{l}325 \\
(93.9)\end{array}$ & $\begin{array}{l}3.52(2.18- \\
5.92, p<0.001)\end{array}$ & $\begin{array}{l}2.86(1.74- \\
4.89, \mathrm{p}<0.001)\end{array}$ \\
\hline & Widowed & $\begin{array}{l}14 \\
(16.9)\end{array}$ & $\begin{array}{l}69 \\
(83.1)\end{array}$ & $\begin{array}{l}1.12(0.62- \\
2.16, p=0.717)\end{array}$ & $\begin{array}{l}1.18(0.63- \\
2.33, p=0.613)\end{array}$ \\
\hline \multirow[t]{5}{*}{ Occupation } & $\begin{array}{l}\text { Neither work nor } \\
\text { study }\end{array}$ & $\begin{array}{l}78 \\
(8.2)\end{array}$ & $\begin{array}{l}876 \\
(91.8)\end{array}$ & - & - \\
\hline & No answer & $\begin{array}{l}1 \\
(14.3)\end{array}$ & $\begin{array}{l}6 \\
(85.7)\end{array}$ & $\begin{array}{l}0.53(0.09- \\
10.16, p= \\
0.564)\end{array}$ & $\begin{array}{l}0.56(0.09- \\
10.97, p= \\
0.602)\end{array}$ \\
\hline & Work & $\begin{array}{l}192 \\
(13.7)\end{array}$ & $\begin{array}{l}1213 \\
(86.3)\end{array}$ & $\begin{array}{l}0.56(0.42- \\
0.74, p<0.001)\end{array}$ & $\begin{array}{l}0.55(0.41- \\
0.73, p<0.001)\end{array}$ \\
\hline & Work and study & $1(5.9)$ & $\begin{array}{l}16 \\
(94.1)\end{array}$ & $\begin{array}{l}1.42(0.28- \\
25.88, p= \\
0.733)\end{array}$ & $\begin{array}{l}1.01(0.19- \\
18.75, p= \\
0.992)\end{array}$ \\
\hline & Study & & $\begin{array}{l}7 \\
(100.0)\end{array}$ & NA & NA \\
\hline \multirow[t]{2}{*}{ HIV_Status } & Negative & $\begin{array}{l}217 \\
(10.2)\end{array}$ & $\begin{array}{l}1908 \\
(89.8)\end{array}$ & - & - \\
\hline & Positive & $\begin{array}{l}55 \\
(20.8)\end{array}$ & $\begin{array}{l}210 \\
(79.2)\end{array}$ & $\begin{array}{l}0.43(0.31- \\
0.61, \mathrm{p}<0.001)\end{array}$ & $\begin{array}{l}0.45(0.32- \\
0.65, \mathrm{p}<0.001)\end{array}$ \\
\hline Years_as_PWID & Mean (SD) & $\begin{array}{l}9.2 \\
(6.0)\end{array}$ & $\begin{array}{l}8.7 \\
(6.4)\end{array}$ & $\begin{array}{l}0.99(0.97- \\
1.01, p=0.154)\end{array}$ & $\begin{array}{l}1.00(0.98- \\
1.02, p=0.945)\end{array}$ \\
\hline \multirow[t]{2}{*}{ Ever_Incarcerated } & No & $\begin{array}{l}183 \\
(10.6)\end{array}$ & $\begin{array}{l}1549 \\
(89.4)\end{array}$ & - & - \\
\hline & Yes & $\begin{array}{l}88 \\
(13.8)\end{array}$ & $\begin{array}{l}551 \\
(86.2)\end{array}$ & $\begin{array}{l}0.74(0.57- \\
0.97, \mathrm{p}=0.030)\end{array}$ & $\begin{array}{l}0.92(0.68- \\
1.26, p=0.605)\end{array}$ \\
\hline
\end{tabular}

Most PWID (88.6\%) reported always having access to clean syringes.

\section{Discussion}


This paper found that methadone maintenance therapy is underutilized among PWID in Tajikistan, and relatively few PWID are registered with the narcology centers. Accessibility and utilization of clean needles and syringes, however, is relatively high.

NSP's are well funded in the country by NGOs and the Tajik government through the trust point system. The high engagement with NSPs may indicate that the integration of NSP with other healthcare and harm reduction services at 'trust points' is an effective way of increasing the use of clean needles among PWID.

Although the incidence of HIV among PWID in Tajikistan is growing, it is not growing nearly as quickly as for those cases transmitted heterosexually (Fig. 1). The high utilization of NSP among PWID, however, is not reflected in the growing rates of new HIV infections among PWID in the country.

Data suggest that an effective way of preventing HIV transmission among PWID is by upscaling opioid agonist therapy (OAT) such as methadone for opioid use disorders. OAT prevents new HIV infection by decreasing the frequency of injection among PWID. The data showed very low engagement with methadone, especially among the general PWID population, indicating that to address growing HIV rates methadone programs will need to be upscaled in Tajikistan. Increasing OST education and counseling, expanding the number of methadone sites across the country, and moving methadone programs out of narcology centers and into an independent network are some of the ways Tajikistan could transform OST programs from a "perpetual pilot" to a more effective and expansive system.

OAT is also shown to increase adherence to ART, therefore, while PWID with HIV are more likely to engage with methadone than the general PWID population, greater work will need to be done to enroll PLHIV in methadone programs as part of their treatment protocol. Low OAT usage in Tajikistan mirrors other settings in the post-Soviet world where social norms and myths impede successful interventions $(14,15)$.

Earlier in this paper we discussed the higher number of NGOs targeting at risk groups among IDUs rather than the general PWID population. This is reflected in the dataset with MSM, PLHIV and formerly incarcerated PWID several times more likely to be registered with a narcology center. Similarly, MSM and PLHIV are more likely to be enrolled in a methadone program than the general PWID population. The increased likelihood of service engagement among at risk PWID groups implies that organizations such as Equal Opportunities and Spin Plus are effective at linking people to care.

Beyond upscaling services for the general PWID population, the Tajik government and NGO's should address the disparity in engagement with healthcare between ethnic Tajiks and ethnic minorities. Ethnic Uzbeks and Ethnic Russians (the first and third largest ethnic minority groups respectively), were less likely to register with a narcology center and enroll in a methadone program than ethnic Tajiks.

Accounting for linguistic and cultural difference in existing services and education programs, creating targeted services for ethnic minorities, and building networks of community educators and healthcare providers are some of the ways the Tajik government and NGO's could work on addressing the gap in service engagement between ethnic Tajiks and ethnic minorities in the country. 
A limitation of this study is that the IBBS survey could have oversampled PLHIV, MSM and formerly incarcerated PWID because they are already engaged with NGO and government healthcare services. PWID in the general population who are not registered or engaged in services may be "harder to reach" for surveying purposes and therefore underrepresented in the survey. Another limitation of this study is exclusion of questions regarding religion and region of origin in the questionnaire. For example, people living with HIV in the Gorno-Badakhshan Autonomous Oblast are more likely to be PWID than any other region in Tajikistan, however, the IBBS does not account for regional difference that could explain the high level of HIV infection among PWID in Gorno-Badakhshan. Future BBS should include questions about region of origin and religion to lend a greater understanding of service utilization among PWID in the country.

\section{List Of Abbreviations}

Bio-behavioral survey (BBS), Opioid agonist therapy (OST), People living with HIV (PLHIV), People who inject drugs (PWID) People who use drugs (PWUD), Men who have sex with men (MSM), Needle and syringe program (NSP).

\section{Declarations}

\section{Ethics approval and consent to participate}

Not applicable. This is a secondary analysis of a de-identified dataset and therefore does not require ethical approval.

\section{Consent for publication}

Not applicable.

\section{Availability of data and materials}

The data that support the findings of this study are the property of the Ministry of Health of Tajikistan and are not publicly available.

\section{Competing interests}

The authors declare that they have no competing interests.

\section{Funding}

No funding was received for any portion of the data collection, treatment of patients, interventions or any other related activities involved in creation of Tajikistan's HIV surveillance system or the system's corresponding dataset. These activities are solely the responsibility of Tajikistan's government and Ministry of Health and receive no funding from outside sources. 
Daniel Bromberg's doctoral studies, and therefore time allocated to this paper, were supported by funding from the United States' National Institute of Mental Health (T32 MH20031). The content is solely the responsibility of the authors and does not necessarily represent the official views of the National Institute of Mental Health or the National Institutes of Health.

\section{Authors' contributions}

AA designed the study. DJB, MMT and AA conducted the statistical analysis and wrote the initial manuscript with substantial input from KA, SK, and DS. All authors approved the final manuscript.

\section{Acknowledgements}

The authors would like to acknowledge the Tajikistan Ministry of Health, Republican AIDS Center, ICAP at Colombia university and USA-CDC for their support in providing the dataset used in this analysis, reviewing several iterations of the manuscript, and general support in conducting this research

\section{References}

1. Malloch-Brown M. Opium production in Afghanistan. BMJ. 2008;336(7651):972-.

2. Drugs UNOo, Asia CROfC, Uzbekistan. Illicit Drug Trends in Central Asia. 2008.

3. Merz F. United Nations Office on Drugs and Crime: World Drug Report 2017. 2017. SIRIUS-Zeitschrift für Strategische Analysen. 2018;2(1):85-6.

4. Latypov A, Otiashvili D, Zule W. Drug scene, drug use and drug-related health consequences and responses in Kulob and Khorog, Tajikistan. Int J Drug Policy. 2014;25(6):1204-14.

5. UNODC Report on Tajikistan. 2010.

6. Elovich R, Drucker E. On drug treatment and social control: Russian narcology's great leap backwards. Harm reduction journal. 2008;5:23-

7. Mansfeld M, Ristola M, Klinte J, Bultman J, Kastelic A, Rotberga S. HIV programme review in Tajikistan. 2015.

8. Program UND. Project Document - Tajikistan. 2015.

9. Latypov A. Opioid substitution therapy in Tajikistan: Another perpetual pilot? International Journal of Drug Policy. 2010;21(5):407-10.

10. EMCDDA. Country overview: Tajikistan. 2014.

11. Rabin BA, Brownson RC. Terminology for dissemination and implementation research. Dissemination and implementation research in health: Translating science to practice. 2017;2:19-45.

12. Harrison E, Drake T, Ots R. finalfit: Quickly Create Elegant Regression Results Tables and Plots when Modelling. R package version 09 0, URL https://CRAN R-project org/package= finalfit. 2019.

13. Allaire J. RStudio: integrated development environment for R. Boston, MA. 2012;770:394. 
14. Polonsky M, Rozanova J, Azbel L, Bachireddy C, Izenberg J, Kiriazova T, et al. Attitudes toward addiction, methadone treatment, and recovery among HIV-infected Ukrainian prisoners who inject drugs: incarceration effects and exploration of mediators. AIDS and Behavior. 2016;20(12):2950-60.

15. Polonsky M, Azbel L, Wickersham JA, Marcus R, Doltu S, Grishaev E, et al. Accessing methadone within Moldovan prisons: prejudice and myths amplified by peers. Int J Drug Policy. 2016;29:91.

\section{Figures}

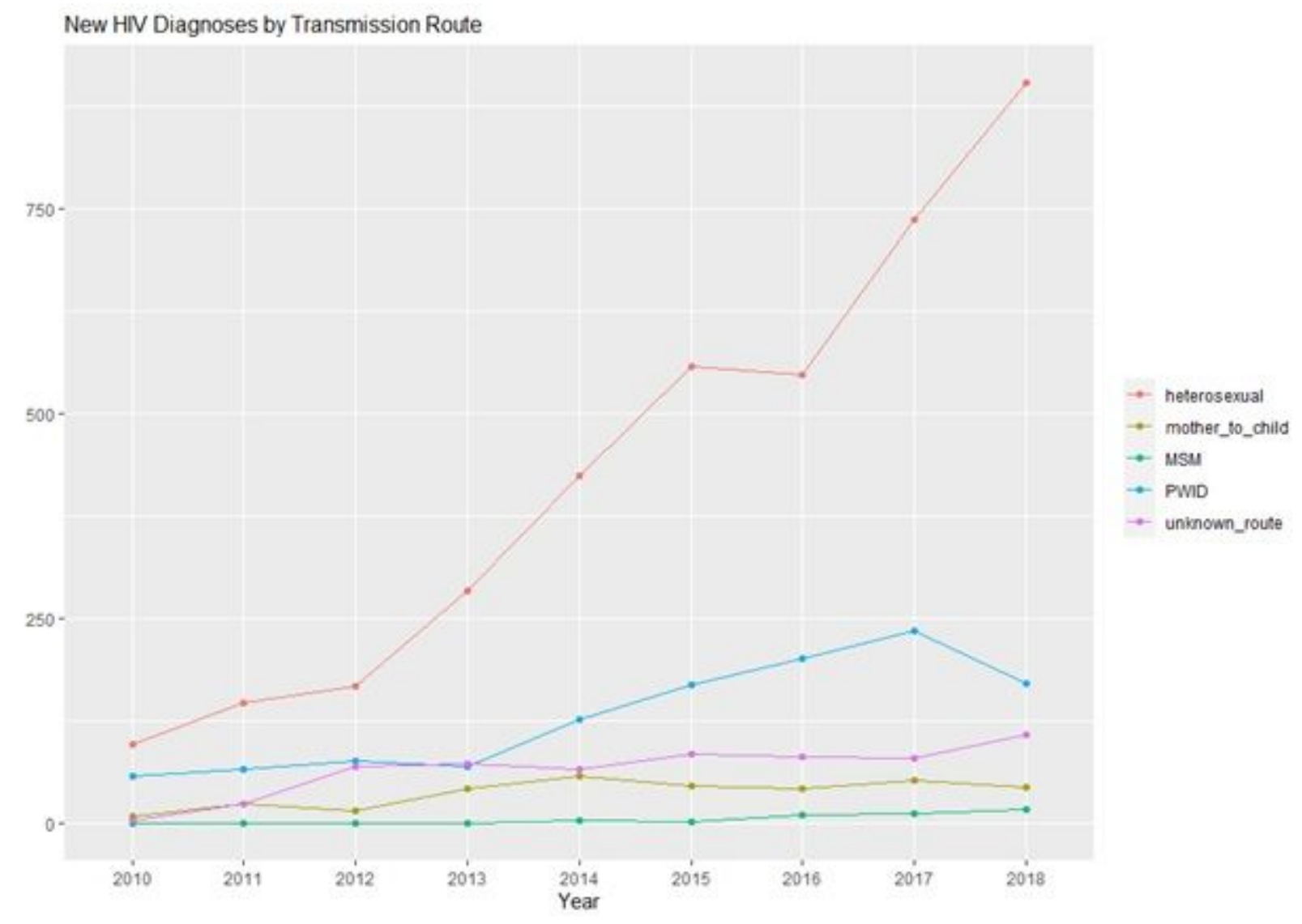

\section{Figure 1}

Incidence of HIV by Transmission Route in Tajikistan. Data from National AIDS Registry 